\title{
Regional Variation in Fracture-Associated Prescription Drug Use and Hip Fractures in Long-Term Care: an Observational Study
}

J Gen Intern Med 36(11):3604-7

DOI: $10.1007 / \mathrm{s} 11606-020-06477-8$

(C) Society of General Internal Medicine 2021

\section{INTRODUCTION}

We previously demonstrated that concurrent exposure to three or more fracture-associated drugs (FADs) increases individual fracture risk up to 4 -fold. ${ }^{1}$ Moreover, intensity of FAD exposure in long-term care (LTC) is twice that of communitydwelling Medicare beneficiaries. ${ }^{2}$ To advance knowledge of the impact of intense prescribing as potential iatrogenic risk, we explore regional variation of prescription drug use intensity and its association with hip fracture rates among LTC residents. We examined intensity of prescription use categorized as total, or FAD and non-FAD in LTC beneficiaries across Hospital Referral Regions (HRRs), and we assess the association between this intensity and hip fracture rates. We hypothesize that measuring variation in prescription intensity can identify regions (and LTC facilities) with relatively high use of concurrent FADs; this may inform interventions aimed at improving health outcomes and safety of this vulnerable population.

\section{METHODS}

\section{Data Sources and Cohort}

We used Medicare Parts A (inpatient) and B (outpatient) administrative data, 2004-2014, and Part D (prescription), 2006-2014, for a $40 \%$ random sample of fully enrolled, feefor-service beneficiaries. ${ }^{1} \mathrm{We}$ identified beneficiaries residing

Question: How much regional variation exists in the intensity of fractureassociated drug use in the long-term care (LTC) setting, and how is this variation associated with fracture risk?

Findings: In this cohort study, 1.8 million person-years of Medicare data revealed substantial variation in receipt of three or more concurrent fracture-associated drugs, among LTC residents; areas with greater use of fracture-associated drugs experienced higher risk-adjusted fracture rates.

Meaning: Guidelines to reduce fracture-associated drug use in LTC settings could decrease fractures in this vulnerable population.

Received August 3, 2020

Accepted December 15, 2020

Published online January 26, 2021 in LTC as those with $50 \%$ or more of annual prescription fills dispensed by a LTC pharmacy. ${ }^{3}$

\section{Hip Fracture and Prescription Drug Receipt}

Hip fractures were identified from inpatient claims using International Classification of Diseases, Ninth Revision (ICD-9) Diagnosis Codes. ${ }^{1}$ Twenty-one distinct FAD groups were assessed including antidepressants, antipsychotics, loop diuretics, proton pump inhibitors (PPIs), and opioids. ${ }^{1}$ Fill records identified medications received, and "days supply" set duration. For each person day, the status for each FAD group was assigned as exposed or not.

\section{Statistical Analysis}

For each HRR ( $n=306)$, we counted receipt of three or more (multiple) total drugs, or categorized as FADs and non-FADs. Descriptive measures of prescription receipt (mean, standard deviation (SD), and range) are presented as \% of total person years (\%PY) observed among LTC beneficiaries. Logistic regression modelled hip fracture risk associated with HRRlevel intensity of multiple drug receipt adjusted for age, sex, and race/ethnicity using SAS software, version 9.4. Fracture risk associated with the highest versus the lowest quintile of population-level drug receipt intensity per HRR is presented as risk ratios (RR) and 95\% confidence intervals (CI). Significant associations were considered at $p$ value $<0.05$.

\section{RESULTS}

We observed 36,409 hip fractures among 1,803,544 person years (422,111 beneficiaries) over 8 years. Across all HRRs, mean \pm SD multiple drug receipt, for total, FADs and nonFADs, characterized $58.9 \pm 3.4,44.0 \pm 3.6$, and $19.4 \pm 3.4$ $\%$ PY observed, respectively. The HRR-level range of multiple FAD receipt was 10.4-28.5 \%PY. Regions with the greatest multiple FAD use intensity were in the southeast; Gulfport and Oxford, MS, Alexandria and Monroe, LA and Miami, FL; low intensity regions were Bronx, NY; Bend, OR; Albuquerque, NM; Honolulu, HI; Sun City, AZ (Fig. 1a, b).

Logistic regression demonstrated modest but significant hip fracture risk associated with HRR-level total drug use intensity, comparing HRRs in the highest versus lowest quintile (OR $=1.13,95 \%$ CI $1.08-1.19, p<0.001)$. In models considering 


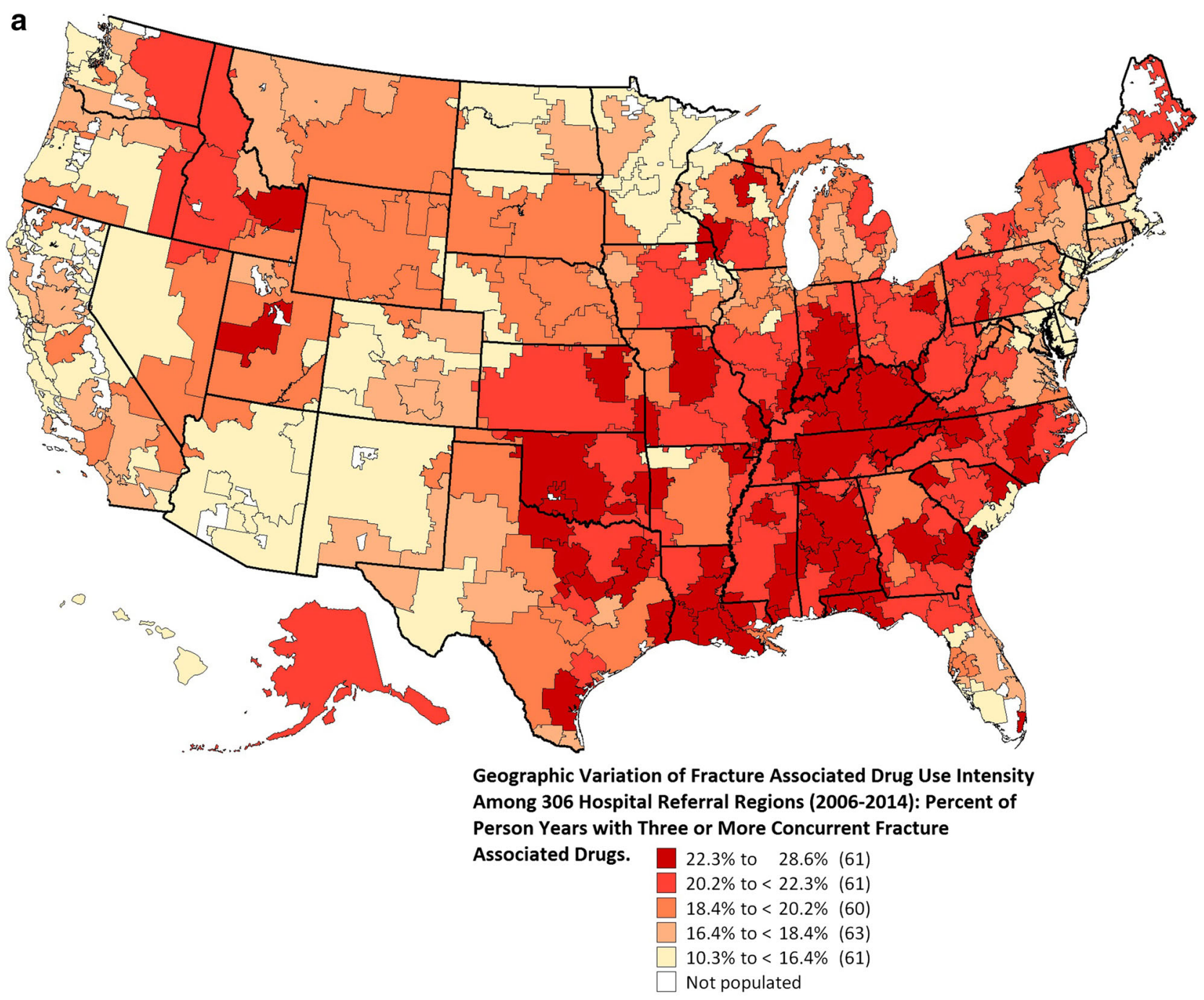

b

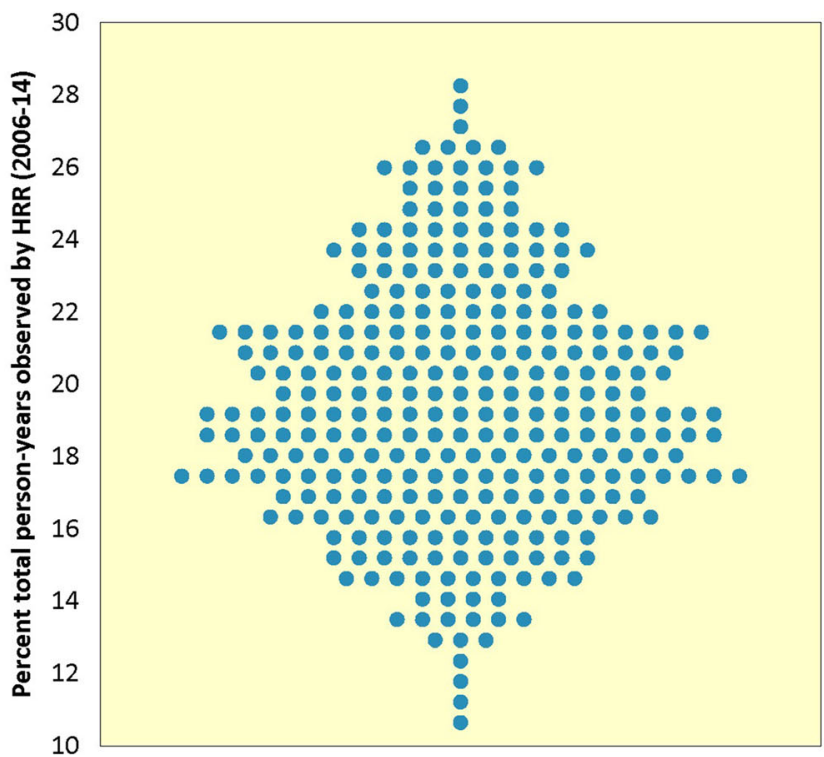

\begin{tabular}{|c|c|}
\hline \multicolumn{2}{|c|}{$\begin{array}{c}\text { Distribution of Fracture Associated Drug Use Intensity Among } \\
\mathbf{3 0 6} \text { Hospital Referral Regions: Percent of Person Years with } \\
\text { Three or More Concurrent Fracture Associated Drugs. }\end{array}$} \\
\hline Hospital Referral Region & $\%$ Exposure \\
\hline \multicolumn{2}{|l|}{5 highest regions } \\
\hline Gulf port, MS & $28.5 \%$ \\
\hline Oxford, MS & $27.7 \%$ \\
\hline Alexandria, LA & $27.3 \%$ \\
\hline Miami, FL & $26.6 \%$ \\
\hline Monroe, LA & $26.6 \%$ \\
\hline \multicolumn{2}{|l|}{5 lowest regions } \\
\hline Bronx, NY & $13.0 \%$ \\
\hline Bend, OR & $12.4 \%$ \\
\hline Albuquerque, NM & $11.5 \%$ \\
\hline Honolulu, $\mathrm{HI}$ & $10.9 \%$ \\
\hline Sun City, AZ & $10.4 \%$ \\
\hline
\end{tabular}

Figure 1 a Geographic variation of fracture-associated drug use intensity in long-term care Medicare beneficiaries across 306 Hospital Referral Regions (2006-2014): percent of person years with three or more concurrent fracture-associated drugs. b Distribution plot of fractureassociated drug use intensity among 306 Hospital Referral Regions: percent of person years with three or more concurrent fracture-associated drugs with highest and lowest ranked regions identified. 


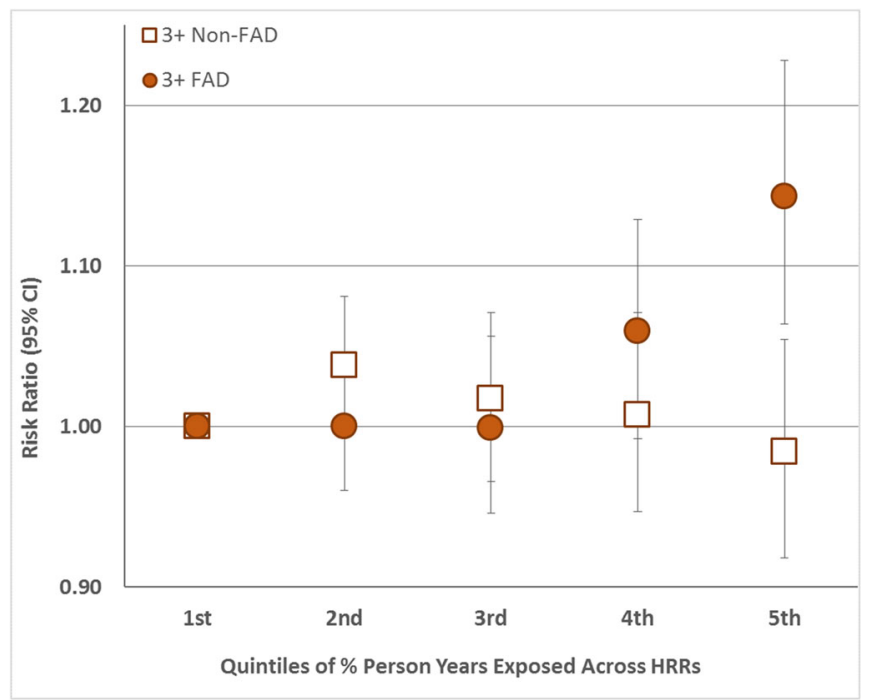

Figure 2 Hip fracture rates among long-term care Medicare beneficiaries residing in 306 Hospital Referral Regions (2006-2014) categorized by quintiles of three of more concurrent drug use intensity (percent of person years exposed) for fracture-associated and non-fracture-associated drugs.

FAD and non-FAD HRR-level intensity (Fig. 2), hip fracture was associated with increased intensity of multiple FADs (5th quintile (ref $=1$ st quintile) $\mathrm{OR}=1.14,95 \%$ CI 1.06-1.23, $p<$ 0.001 ), but not with multiple non-FAD intensity ( $\mathrm{OR}=0.98$, 95\% CI 0.92-1.05, $p=0.20$ ).

We explored specific FADs in regions with high FAD intensity. Compared to beneficiaries residing in HRRs with the lowest multiple FAD receipt rates (1st quintile), those in highest multiple FAD rate HRRs (5th quintile) experienced higher rates of opioids (6.5 vs. $11.8 \%$ PY), PPIs (13.1 vs. 17.2 $\% \mathrm{PY}$ ), loop diuretics (12.7 vs. $16.7 \% \mathrm{PY}$ ), and SSRIs (17.0 vs $22.2 \% \mathrm{PY}$ ), respectively. The latter three FADs were common contributors to 2-way concurrent hip fracture risk in this population. $^{2}$

\section{DISCUSSION}

Among Medicare beneficiaries, FAD use in LTC setting varies substantially across US regions, and higher use is associated with hip fracture risk. These findings should prompt exploration of the drivers of both high and low intensity prescribing and should inform development of interventions aimed at maximizing prescribing quality and minimizing harm potentially conferred by FADs.

Use of administrative data has limitations; prescription fills are a proxy for exposure and we cannot assess clinical appropriateness of prescribing. Nevertheless, we provide evidence supporting guidelines to reduce fracture-associated drug use in LTC settings to decrease fractures in this vulnerable population. $^{4}$
Acknowledgments: The authors wish to thank Atlas Editor, Kristen Bronner, MA, for graphical expertise and Professor Jonathan Skinner, $\mathrm{PhD}$, for review of early manuscript drafts.

Rebecca T. Emeny, $\mathrm{Ph} \mathrm{D}^{1}$

John A. Batsis, $M D^{2}$

Nancy E. Morden, $M D^{3}$

${ }^{1}$ The Dartmouth Institute for Health Policy \& Clinical Practice and Department of Psychiatry, The Geisel School of Medicine at Dartmouth,

Lebanon, NH, USA

${ }^{2}$ Division of Geriatric Medicine, School of Medicine, Department of Nutrition, Gillings School of Global Public Health, University of North Carolina at Chapel Hill,

Chapel Hill, NC, USA

${ }^{3}$ The Dartmouth Institute for Health Policy \& Clinical Practice, The Geisel School of Medicine at Dartmouth, Lebanon, NH, USA

Corresponding Author: Rebecca T. Emeny, PhD; The Dartmouth Institute for Health Policy \& Clinical Practice and Department of Psychiatry, The Geisel School of Medicine at Dartmouth, Lebanon, NH, USA (e-mail: rebecca.t.emeny@dartmouth.edu).

Funding RTE and NEM were supported by the National Institute on Aging: P01 AG019783, Skinner (PI): Causes and Consequences of Healthcare Efficiency. JB was funded in part by the National Institute on Aging of the National Institutes of Health under Award Number K23AG051681.

\section{Compliance with Ethical Standards:}

The Dartmouth Protection of Human Subjects Committee approved this study. 
Conflict of Interest: The authors declare that they do not have a conflict of interest.

Disclaimer: The views expressed in this article are those of the authors and no endorsement by the NIA or the NIH is intended or should be inferred.

\section{REFERENCES}

1. Emeny RT, Chang $\mathbf{C H}$, Skinner J, et al. Association of Receiving Multiple, Concurrent Fracture-Associated Drugs With Hip Fracture Risk. JAMA Netw Open. 2019;2(11):e1915348.

2. Emeny RT, Batsis JA, Morden NE. Intense Use of Fracture-Associated Drugs Among Medicare Beneficiaries in Long-term Care. J General Intern Med. 2020.
3. Harris-Kojetin L, Sengupta M, Park-Lee E, et al. Long-term care providers and services users in the United States:Data from the National Study of Long-Term Care Providers, 2013-2014. Natl Center Health Stat Vital Health Stat. 2016;3(38).

4. By the American Geriatrics Society Beers Criteria Update Expert P. American Geriatrics Society 2019 Updated AGS Beers Criteria(R) for Potentially Inappropriate Medication Use in Older Adults. J Am Geriatr Soc. 2019;67(4):674-694.

Publisher's Note: Springer Nature remains neutral with regard to jurisdictional claims in published maps and institutional affiliations. 